\title{
ON THE DAMPING OF WATER HYDRAULIC CYLINDER DRIVES
}

\author{
Tapio VIRVALO*, Esa MÄKINEN** and Matti VILENIUS** \\ * IHA, Tampere University of Technology \\ P.O.Box 589, 33101 Tampere, Finland \\ Fax int-358-3-365240, e-mail virvalo@cc.tut.fi, makinen@cc.tut.fi \\ ** IHA, Tampere University of Technology \\ P.O.Box 589, 33101 Tampere, Finland
}

\begin{abstract}
The main aim when designing a controller for a demanding water hydraulic servo application is to find a good and practical method for increasing system damping. The low natural damping of water hydraulic cylinder drives has to be carefully considered also in open loop control applications. The damping of a cylinder drive depends on many factors. Traditionally, mainly based on the linear model, damping is considered to result from viscous friction force. However, if viscous friction force were the only factor achiving damping of a system, it should have to be unpractically great. In this paper the effects of the main factors influencing the damping of water hydraulic cylinder drives are studied on the basis of experimental data and simulation. The individual effects of these factors are studied by comparing simulation results with experimental open loop velocity and pressure measurements.
\end{abstract}

\section{KEYWORDS}

Water hydraulic cylinders, water hydraulic servo drives

\section{NOMENCLATURE}

$A_{\text {pis }} \quad$ Average piston area $\left[\mathrm{m}^{2}\right]$

$\mathrm{B}_{\text {eff }}$ Effective bulk modulus [Pa]

$\mathrm{Kp}$ Pressure gain of valve $[\mathrm{Pa} / \mathrm{V}]$

$\mathrm{K}_{\mathrm{qa}} \quad$ Velocity gain $\left[\mathrm{m} /\left(\mathrm{s}^{*} \mathrm{~V}\right)\right]$

$\mathrm{L}$ Stroke length $[\mathrm{m}]$

lea Leakage percentage of valve [\%]

$\mathrm{M} \quad$ Effective inertia load [kg]

$\mathrm{p}_{\mathrm{sN}} \quad$ Nominal supply pressure (the leakage of the valve has been specified with it) $[\mathrm{Pa}]$

$Q_{N} \quad$ Nominal volume flow of valve $\left[\mathrm{m}^{3} / \mathrm{s}\right.$ ]

s Laplace operator

$\mathrm{u} \quad$ Control signal of valve [V]

$u_{\max } \quad$ Maximum control signal of valve [V]

$\mathrm{v}$ Velocity of piston $[\mathrm{m} / \mathrm{s}]$

$\mathrm{V}_{\text {tot }}$ Total water volume $\left[\mathrm{m}^{3}\right]$

\author{
$\mathrm{V}_{10} \quad$ Dead volume of piston side $\left[\mathrm{m}^{3}\right]$ \\ $\mathrm{V}_{20} \quad$ Dead volume of rod side $\left[\mathrm{m}^{3}\right]$ \\ $y \quad$ Position of piston [m] \\ $\omega_{n} \quad$ Natural frequency $[\mathrm{rad} / \mathrm{s}]$ \\ $\delta_{n} \quad$ Damping factor [-] \\ $\Delta \mathrm{p} \quad$ Pressure drop in valve notch [Pa] \\ $\Delta \mathrm{p}_{\mathrm{N}} \quad$ Nominal pressure drop of valve [Pa]
}

\section{INTRODUCTION}

$2^{\text {nd }}$ - and $3^{\text {rd }}$-order linear models are used in the design of the steering and control of a water hydraulic cylinder drive. The $2^{\text {nd }}$-order model, Eq.(1), is used between the velocity of a piston and the control signal of a valve. The $3^{\text {rd }}$-order model, Eq.(2) is used between the position of a piston and the control signal of a valve. The dynamics of a servo valve is assumed to be significantly higher than the natural 
frequency of a cylinder drive.

$$
\begin{gathered}
\frac{V(s)}{U(s)}=\frac{K_{q a} * \omega_{n}^{2}}{s^{2}+2 * \delta_{n} * \omega_{n} * s+\omega_{n}^{2}} \\
\frac{Y(s)}{U(s)}=\frac{K_{q a} * \omega_{n}^{2}}{s *\left(s^{2}+2 * \delta_{n} * \omega_{n} * s+\omega_{n}^{2}\right)}
\end{gathered}
$$

These models are based on the assumption that the dynamics of a control valve is significantly higher (3...5 times) than the dynamics of a cylinder. Both models have three parameters that have to be evaluated according to the specifications of an application, Figure 1.

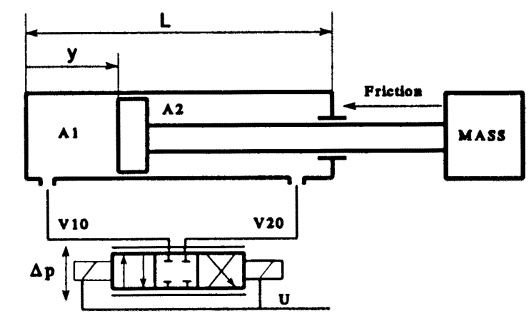

Figure 1 Valve controlled cylinder

The velocity gain depends on the nominal flow rate of a valve, the ratio of actual pressure drop in a valve to the nominal pressure drop as well as the maximum control signal of a valve and the average piston area, according to Eq.(3).

$$
K_{q a}=\frac{Q_{N} * \sqrt{\frac{\Delta p}{\Delta p_{N}}}}{A_{p i s} * u_{\max }}
$$

The actual natural frequency depends on the position of the piston, but usually the minimum natural frequency is used in the preliminary design. The minimum natural frequency is calculated with Eq.(4) (the denotations in Figure 1).

$$
\omega_{n \min }=\sqrt{\frac{B_{e f f} *\left(\sqrt{A_{1}}+\sqrt{A_{2}}\right)^{2}}{\left(L+\frac{V_{10}}{A_{1}}+\frac{V_{20}}{A_{2}}\right) * M}}
$$

The natural damping factor of the linear model of a cylinder can be evaluated according to Eq.(5).

$$
\delta_{n}=\frac{K_{c e}}{A_{p t s}} * \sqrt{\frac{B_{e f f} * M}{V_{t o t}}}+\frac{b}{4 * A_{p i s}} * \sqrt{\frac{V_{t o t}}{B_{e f f} * M}}
$$

In this equation there are parameters that can be evaluated quite easily, such as the effective bulk modulus, the total volume, the effective inertia load and the average piston area. Two other parameters, $\mathbf{K}_{\mathrm{ce}}$ and $\mathbf{b}$, cannot be defined so easily. The parameter $\mathbf{K}_{\mathrm{ce}}$ includes the leakages of a cylinder and the flow-pressure coefficient of a valve, which depends on the leakage of a valve. The parameter $\mathbf{b}$ describes the viscous friction of a cylinder.

\section{THE INFLUENCE OF PARAMETERS ON THE EFFECTIVE DAMPING}

According to Eq.(5) many parameters have an influence on the effective damping of a cylinder drive. These influences are studied in the system with the following specifications: $\checkmark$ Cylinder 63/36-500

$\sqrt{ }$ Nominal inertia load $850 \mathrm{~kg}$

$\checkmark$ Servo valve

* Nominal volume flow $19 \mathrm{1} / \mathrm{min}$ with nominal pressure drop $3.5 \mathrm{MPa} /$ control notch

* Linear volume flow characteristics

* Null position leakage $1 \mathrm{l} / \mathrm{min}$ with 70 bar supply pressure. (The leakage is $5 \%$ of the nominal volume flow)

$\checkmark$ Supply pressure $8.3 \mathrm{MPa}$

$\sqrt{ }$ Pipes between the valve and cylinder, $\mathrm{d}_{\text {in }}=12 \mathrm{~mm}$, length $350 \mathrm{~mm}$ each

According to the specifications above the following data can be evaluated:

$\checkmark$ Effective bulk modulus $\mathrm{B}_{\text {eff }}=2.1 * 10^{9} \mathrm{~Pa}$

$\checkmark$ Total volume $1.464^{*} 10^{-3} \mathrm{~m}^{3}$

$\checkmark$ Average piston area $26.1^{*} 10^{-4} \mathrm{~m}^{2}$

$\sqrt{ }$ Total nominal inertia load $880 \mathrm{~kg}$

With these parameter values the following equation Eq.(6) is achieved for the damping factor:

$$
\delta_{n}=K_{c e} * 1.12 * 10^{10}+b * 3.31 * 10^{-6}
$$

The following units have to be used: $\mathbf{K}_{\mathbf{c e}}\left[\mathbf{m}^{3} /\left(\mathbf{s}^{\star} \mathbf{P a}\right)\right]$ and $\mathbf{b}$ $\left[\mathbf{N}^{*} \mathbf{s} / \mathbf{m}\right]$.

The leakages of a typical commercial water hydraulic cylinder are practically zero. In this situation the parameter $\mathbf{K}_{\mathbf{c e}}$ can be evaluated according to Eq.(7).

$$
\begin{gathered}
K_{c e}=\frac{K_{q}}{K_{p}} \\
K_{c e}=\frac{l e a * Q_{N}}{100 * p_{s N}}
\end{gathered}
$$

The numeric value of $\mathrm{K}_{\mathrm{cc}}$ with the specified valve is $2.26^{*} 10^{-12}\left[\mathrm{~m}^{3} / \mathrm{s} * \mathrm{~Pa}\right]$.

With practical parameter values the first term of Eq.(5) and Eq.(6) has a greater influence on the damping factor than the second term of the equation. This means that the damping factor grows when the inertia load increases. The damping factor grows also when both the size of the servo valve and the leakage of the servo valve increase. 
According to Eq.(5), $\mathrm{Eq}(6)$ and Eq.(7) the damping factor is 0.13 when the leakage of the valve is $5 \%$ (a reasonable value) and the viscous friction coefficient is $30000 \mathrm{~N}^{*} \mathrm{~s} / \mathrm{m}$ (an unpractically high value).

\section{EXPERIMENTAL TESTS}

The following experimental tests were carried out for the cylinder drive studied:

$\checkmark$ Open loop responses with the different valve control signals.

$\checkmark$ Piston velocity and chamber pressures were recorded

The open loop velocity response is used to identify the natural frequency and the damping factor. The result of the identification with the low control signal is depicted in Figures 2 and 3.

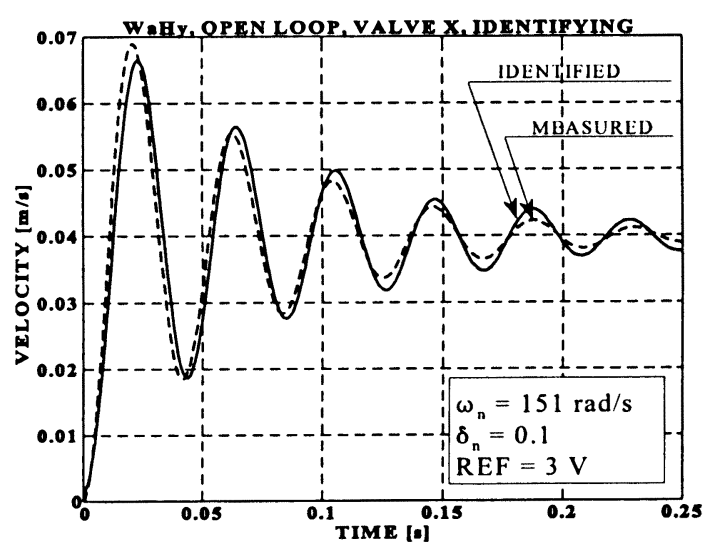

Figure 2 Velocity step responses at the beginning of the stroke, measured and calculated with the identified linear model.

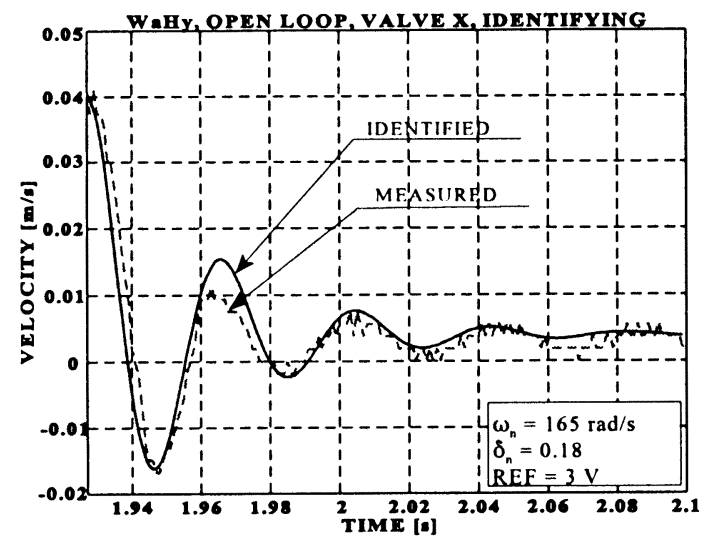

Figure 3 Velocity step responses at the end of the stroke, measured and calculated with the identified linear model. The offset of the valve is $1.5 \%$.

The identified natural frequency, about $151 \ldots 165 \mathrm{rad} / \mathrm{s}$, corresponds quite well to that calculated, $160 \mathrm{rad} / \mathrm{s}$. The identified damping factor, $0.1 \ldots 0.18$, is significantly higher than the linear model shows.

Measured responses also show clearly that at the beginning of responses the damping factor depends strongly on the value of the control signal of a valve. Higher control signals cause higher damping factors. This indicates that the flow-pressure gain of a valve has a significant effect on the damping factor.

When the piston moves with a constant velocity without an external load force, only the viscous friction and Coulomb friction forces influence the piston. The following assumption can be made without any significant error:

$\checkmark$ The viscous friction force depends linearly on the piston velocity

$\checkmark$ The Coulomb friction force does not depend on the piston velocity

On the basis of the measurements and the above assumptions the viscous friction force is depicted in Figure 4 as a function of the piston velocity.

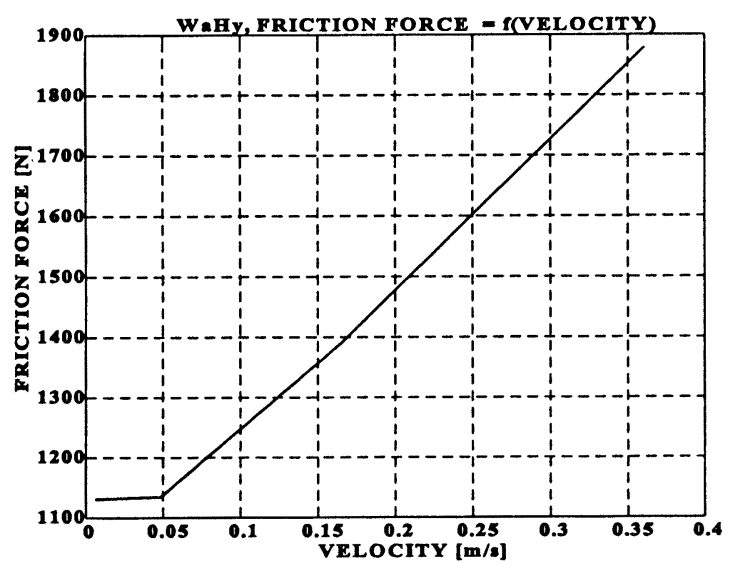

Figure 4 The total friction force as a function of the velocity in outward movement, measured result.

According to Figure 4 the average viscous friction coefficient $b$ is $\mathbf{2 4 0 0} \mathbf{N}^{*} \mathbf{s} / \mathbf{m}$. This is quite a low viscous friction coefficient as far as the damping factor is concerned. Also the Coulomb friction force can be evaluated on the basis of Figure 4. The Coulomb friction force is about $1050 \mathrm{~N}$.

\section{SIMULATION RESULTS}

Both the leakage percentage of the valve and the viscous friction coefficient have to be unrealistically high to achieve the actual damping factor of the linear model of a cylinder drive. Later on the open loop simulation results (solid line) are compared with the measured open loop responses (dashed line). The piston velocity and cylinder chamber pressure responses are used in comparisons. In the nonlinear simulation model the influence of the following parameters is studied:

$\checkmark$ The viscous friction coefficient

$\checkmark$ The leakage percentage of a valve

$\checkmark$ The Coulomb friction force

$\sqrt{ }$ The zero point offset of a valve 


\section{$\sqrt{ }$ The over/under laps of valve control notches}

Every parameter is varied in a certain range and the simulation results and the measured responses are compared. The comparison is made by plotting both responses in the same figure. The simulation is made with the wellverified nonlinear model [1], [2] and [3]. The following characteristics are compared:

$\sqrt{ }$ The shape of responses of the velocity and pressures

$\sqrt{ }$ The steady state values of the velocity and pressures

$\sqrt{ }$ The dynamic behaviour of the velocity and pressures

\section{The influence of the viscous friction coefficient on damping}

The influence of the viscous friction coefficient is studied using the following parameter values:

$\sqrt{ }$ The viscous friction coefficient 2400 and $30000 \mathrm{~N}$ s $/ \mathrm{m}$

$\checkmark$ The Coulomb friction force $\mathbf{F}_{\mathrm{ef}}=\mathbf{0}$

$\checkmark$ The leakage percentage of the valve lea $=0$

$\sqrt{ }$ All offsets and laps of the valve are zero

$\sqrt{ }$ Supply pressure $\mathbf{p}_{\mathbf{s}}=8.3 \mathrm{MPa}$

$\sqrt{ }$ The control signal of the valve REF $=\mathbf{3} \mathbf{V}$

- The constant piston velocity is about $0.04 \mathrm{~m} / \mathrm{s}$

The velocity response is shown in Figure 5 when the viscous friction coefficient value of $2400 \mathrm{~N}^{*} \mathrm{~m} / \mathrm{s}$ is used.

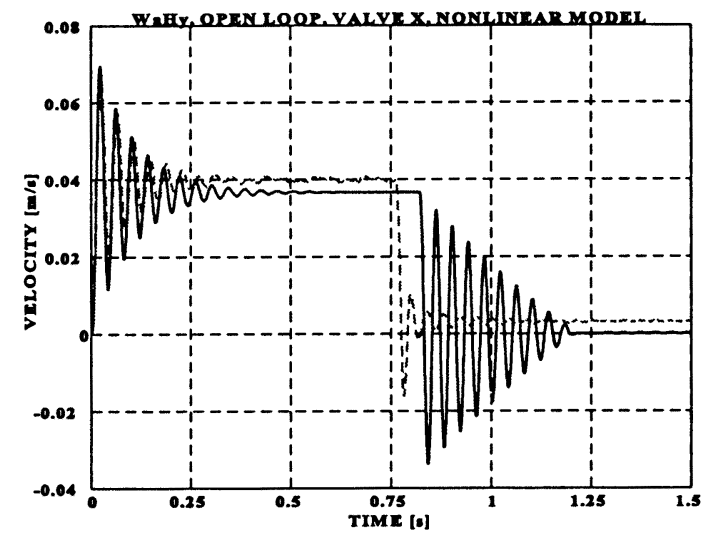

Figure 5 Measured and simulated velocity responses. The viscous friction coefficient is $2400 \mathrm{~N} * \mathrm{~m} / \mathrm{s}$, the leakage and Coulomb friction force are zero and the offset is zero.

The response is very oscillating, indicating that the damping is far too low. At the beginning of the response the damping matches quite well, because of the effect of the opening of the valve.

In Figure 6 the viscous friction coefficient is increased to $30000 \mathrm{~N}^{*} \mathrm{~s} / \mathrm{m}$ and other parameters are kept unchanged. The damping has been increased, but the constant velocity has decreased further. However, the viscous friction coefficient is unrealistically high. This shows that the viscous friction does not explain the damping factor of the linear model.

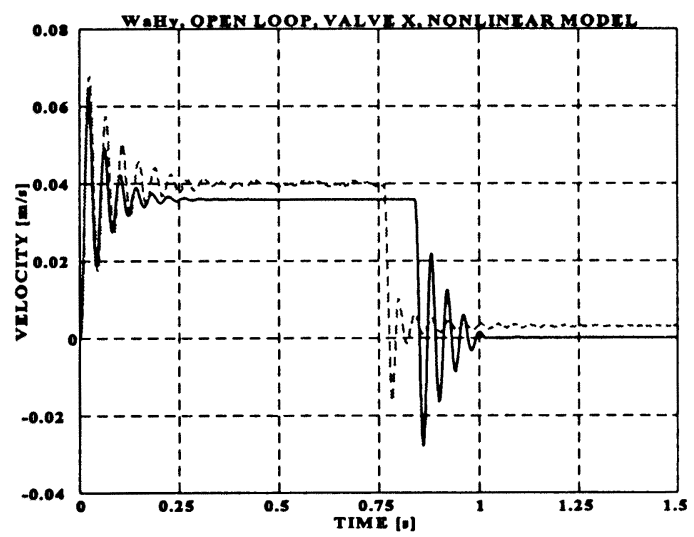

Figure 6 Measured and simulated velocity responses. The viscous friction coefficient is $30000 \mathrm{~N}^{*} \mathrm{~m} / \mathrm{s}$, the leakage and Coulomb friction force are zero and the offset is zero.

\section{The influence of the valve leakage on damping}

Leakages in a cylinder and a valve have an influence on the damping of a cylinder drive, Eq.(6). It can be assumed that the leakages of a normal standard water hydraulic cylinder are zero. In spool valves some leakage always occurs, especially in valves with a zero overlap.

In order to study the influence of the leakages of a valve on the damping of a cylinder drive, the following simulations were carried out:

$\sqrt{ }$ Viscous friction coefficient is $2400 \mathbf{N}^{*} \mathbf{s} / \mathbf{m}$ and the Coulomb friction force is zero

$\sqrt{ }$ The studied leakage percentage from the nominal flow rate of a valve are 1,5 and 10 with the supply pressure $8.3 \mathrm{MPa}$

The simulation results and the measured result are compared by plotting them into the same figures. The control signal of the valve is $3 \mathrm{~V}$ in every case.

The measured and simulated velocity responses are shown in Figure 7, when the valve leakage percentage of 5 is used

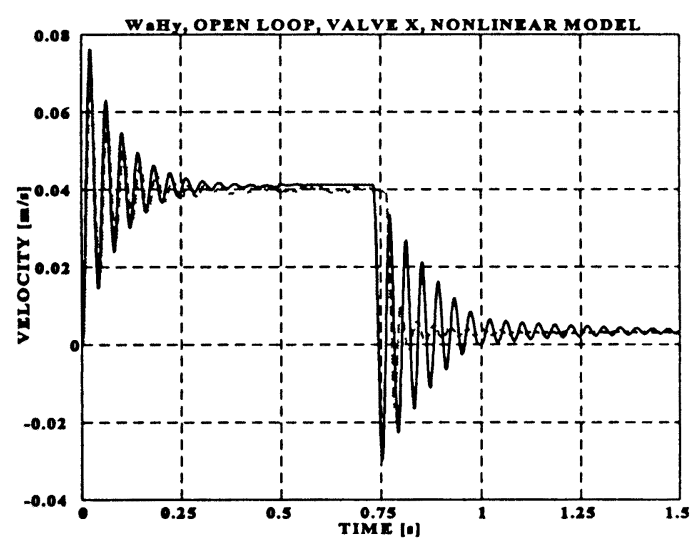

Figure 7 Measured and simulated velocity responses. The viscous friction coefficient is $2400 \mathrm{~N}^{*} \mathrm{~m} / \mathrm{s}$, the leakage percent is $5 \%$, Coulomb friction force is zero and the offset is $1.5 \%$. 
in the simulation. The result is shown in Figure 8, when the

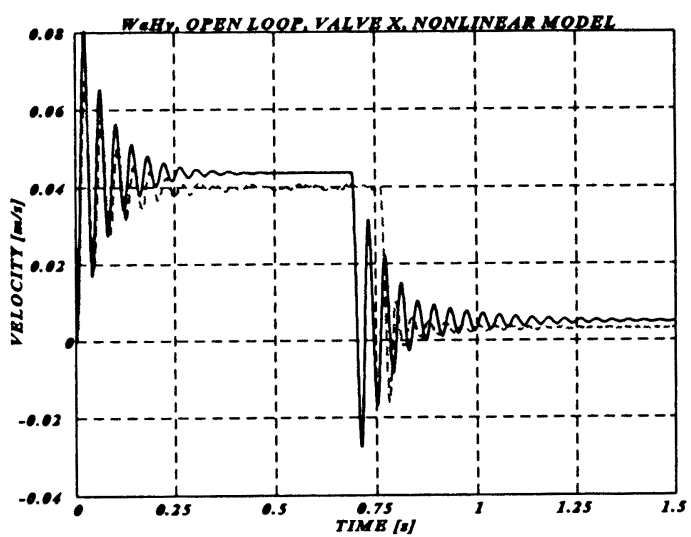

Figure 8 Measured and simulated velocity responses. The viscous friction coefficient is $2400 \mathrm{~N} * \mathrm{~m} / \mathrm{s}$, the leakage percent is $10 \%$, Coulomb friction force is zero and the offset is $1.5 \%$.

leakage percentage is $10 \%$. The steady state values correspond well to each other (the velocity is higher with the higher leakage), but the damping is still too low.

According to Figures $5 \ldots 8$ it is clear that some additional parameters are required to explain the damping in measured responses. The offset of $1.5 \%$ in the simulation corrects the simulated steady state velocity onto the right level.

\section{The influence of friction force on damping}

According to the linear model of a cylinder drive the viscous friction coefficient and valve leakage are the only parameters influencing the damping of a specified cylinder drive. Since only very extreme values of the viscous friction coefficient and valve leakage achieve a suitable damping level in the simulation model, there have to be some nonlinear effects to increase the damping of a cylinder drive. Energy must be consumed to overcome friction forces, which also cause some damping effects. To study the effect of the friction force on a cylinder drive some simulation results are presented.

The velocity responses are depicted in Figure 9. In the simulation the leakage percentage of a valve is $0 \%$, the viscous friction coefficient is $2400 \mathrm{~N}^{*} \mathrm{~s} / \mathrm{m}$ and both the static and the Coulomb friction forces are modelled. The friction forces are modelled according to Eq.(8)

$$
\begin{aligned}
& F_{\text {ast }}=[((1+\operatorname{prco} * p 1)+(2+2 * \operatorname{prco} * p 2)) / 3] * F_{s t} \\
& F_{a c o}=[((1+\operatorname{prco} * p 1)+(2+2 * \operatorname{prco} * p 2)) / 3] * F_{c o}
\end{aligned}
$$

The actual values of both friction forces depend on the pressures in the cylinder chambers. In this case the following values are used:

$\sqrt{ }$ The static friction force $\mathrm{F}_{\mathrm{st}}=1200 \mathrm{~N}$

$\checkmark$ The Coulomb friction force $\mathrm{F}_{\mathrm{co}}=1000 \mathrm{~N}$

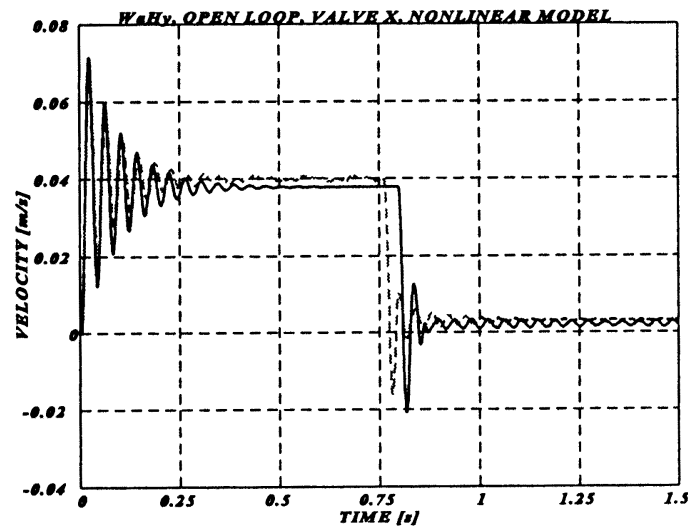

Figure 9 Measured and simulated velocity responses. The leakage is zero and the viscous friction coefficient is 2400 $\mathrm{N}^{*} \mathrm{~s} / \mathrm{m}$. Static and Coulomb friction forces are included. The offset is $1.5 \%$.

$\sqrt{ }$ The pressure coefficient $\operatorname{prco}=5^{*} 10^{-5}$

When the results in Figures 5...9 are compared, the effect of the Coulomb friction force on the damping of a cylinder drive is obvious. The damping in Figure 9 is at the right level.

With the modelled Coulomb friction force the damping of the model and the damping of the real system match well. The Coulomb friction force has almost the same effect on the damping of a cylinder drive as the very high viscous friction coefficient has. The valve leakage is added in order to make the model more realistic .

\section{The influence of the valve spool on damping}

In the detailed modelling of a servo valve, many nonlinearities have to be included in order to enable the simulated and measured cylinder chamber pressures to match [2]. The response of the piston side chamber pressure is shown in Figure 10, when the static characteristics of the valve are linear and the valve leakage is zero.

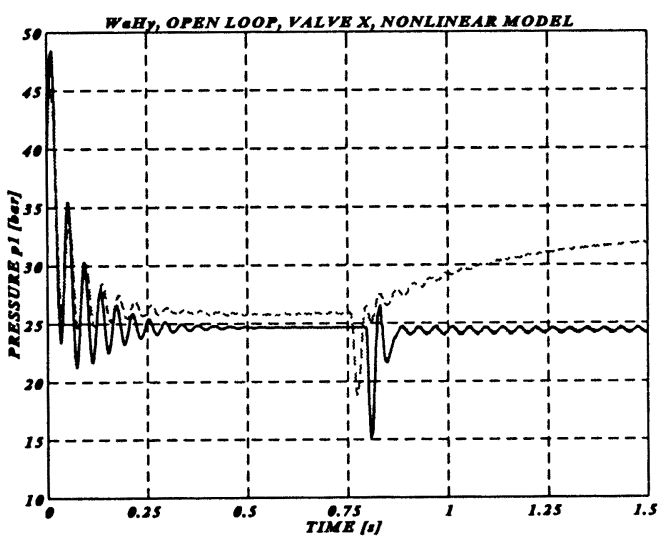

Figure 10 The pressure $\mathrm{p} 1$ response. The viscous friction coefficient is $2400 \mathrm{~N}: \mathrm{s} / \mathrm{m}$. The static and Coloumb friction forces are included. Valve leakage is zero. The offset is $1.5 \%$. 
The only way to influence the chamber pressure level in the steady state is to model also an individual over/under lap in every control notch of a valve. It is obvious that the over lap in the supply side and the under lap in the tank side reduce the chamber pressure level and vice versa.

The response of the piston side pressure and the response of the velocity are depicted in Figure 11 and Figure 12, respectively.

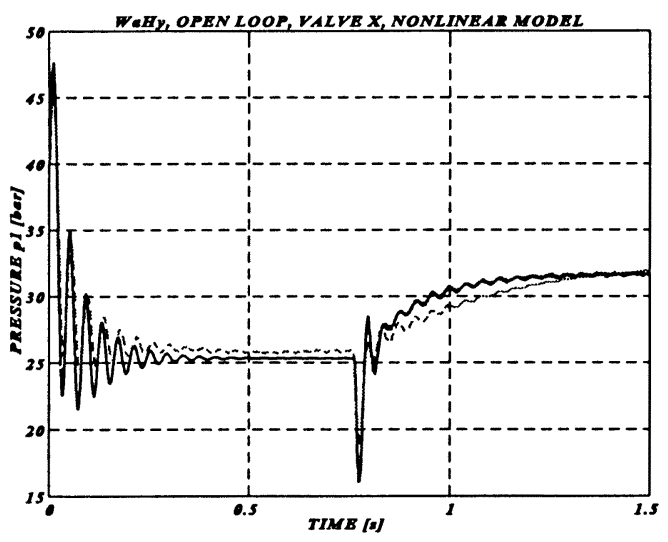

Figure 11 The pressure $\mathrm{p} 1$ response. The viscous friction coefficient is $2400 \mathrm{~N}: \mathrm{s} / \mathrm{m}$. The static and Coloumb friction forces are included. Valve leakage is $5 \%$. The offset is $1.5 \%$ and the individual laps are used in the notches

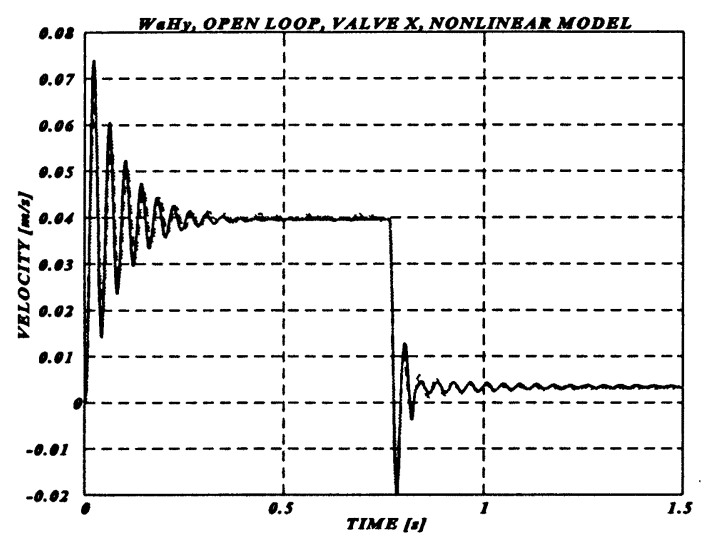

Figure 12 The velocity response. The viscous friction coefficient is $2400 \mathrm{~N}: \mathrm{s} / \mathrm{m}$. The static and Coloumb friction forces are included. Valve leakage is $5 \%$. The offset is $1.5 \%$ and the individual laps are used in the notches.

The static and dynamic characteristics of the simulated and measured responses match well. The viscous friction coefficient, the Coulomb friction force and the leakage of the valve define the effective damping factor of a cylinder drive. The details of the valve control notches define together with the valve leakage the behaviour of the cylinder chamber pressures.

\section{CONCLUSIONS}

When the linear model of a hydraulic cylinder drive is used in the controller design of a servo system, the damping factor should be evaluated. If the physical properties of influencing parameters of the linear model are used in the evaluation of the damping factor, abnormally high numerical values have to be used for the parameters in question. According to this study the following conclusions can be made:

$\checkmark$ The viscous friction force influences the damping factor, but its influence is quite small. Its part is considerably less than half of the actual damping factor.

$\checkmark$ Also the leakage of a control valve influences the damping factor. Its influence depends strongly on the quality of a valve, but in many cases the influence of the valve leakage is about the same as the influence of the viscous friction force.

$\sqrt{ }$ The Coulomb friction force has quite a strong influence on the damping factor. Its influence might be even as high as half of the actual damping factor.

All this means that the damping factor for the linear models of water hydraulic cylinder drives has to be evaluated without any actual connection with the numerical values of physical parameters included in linear models.

\section{REFERENCES}

1. Virvalo, T. Modelling hydraulic position servo realized with commercial components. 3rd International Conference on Fluid Power Transmission and Control, Zhejiang University, Hangzhou China, 13-16 September 1993. 6 p.

2. Virvalo, T. Nonlinear model of analog valve. The Fifth Scandinavian International Conference on Fluid Power, SICFP 97, Linköping, Sweden, 28-30 May 1997. pp. 199-214.

3. Virvalo, T. On the damping of a hydraulic cylinder drive. SICFP'99, May 26-28, 1999, 20 p.

\section{APPENDIX A}

The simulation results show that the Coulomb friction force has a considerable effect on the damping of a cylinder drive. One way to explain this is to consider the actual effect of the Coulomb friction force as somehow similar to the effect of viscous friction. Quillon [A] has presented the idea that the Coulomb friction force can be replaced by the equivalent viscous friction so that the work done during oscillations is the same. Quillon presented the following equation Eq.(A1):

$$
\delta_{c n}=\frac{2}{\pi} * \frac{F_{c}}{K_{h} * z_{0}}
$$

In this example case $\delta_{c n}=0.08$. This value is considerably higher than the value caused by the viscous friction coefficient.

A1 Guillon, M., Hydraulic Servo Systems, Analysis and Design, Butterworths, 1969, 462 p. 